\title{
О кинетике потока жидкости в центробежном сепараторе
}

\author{
Славянский Анатолий Анатольевич \\ ФГБОУ ВО «Московский государственный университет технологий и \\ управления имени К.Г. Разумовского (Первый казачий университет)» \\ Адрес: 109004, город Москва, ул. Земляной Вал, дом 73 \\ E-mail: slavyanskiyaa@mgutm.ru
}

Семенов Евгений Владимирович ФГБОУ ВО «Московский государственный университет технологий и управления имени К.Г. Разумовского (Первый казачий университет)» Адрес: 109004, город Москва, ул. Земляной Вал, дом 73 E-mail:sem-post@mail.ru

Грибкова Вера Анатольевна ФГБОУ ВО «Московский государственный университет технологий и управления имени К.Г. Разумовского (Первый казачий университет)» Адрес: 109004, город Москва, ул. Земляной Вал, дом 73 E-mail:vera_gribkova@list.ru

Николаева Наталья Валерьевна ФГБОУ ВО «Московский государственный университет технологий и управления имени К.Г. Разумовского (Первый казачий университет)» Адрес: 109004, город Москва, ул. Земляной Вал, дом 73 E-mail:nata_nik@inbox.ru

\begin{abstract}
Особенности режимов течения вязкой несжимаемой жидкости в полости между двумя вращающимися конусами имеют большое значение в связи с важностью проблемы анализа особенностей кинетики этих потоков для центробежного сепарирующего оборудования в молочном, мясном, микробиологическом и других смежных производствах. Очевидно, что для исследования кинетических закономерностей движения жидкости в узком зазоре между двумя соседними усеченными конусами необходимо располагать корректным аппаратом математического моделирования течения в этом зазоре, имея в виду, что индуцируемый линейным источником на оси вращения ротора поток приближенно моделирует течение слабо концентрированной системы «жидкость + твердое» в щелевой полости между тарелками сепаратора. В данной работе поставлена цель: на основе законов сохранения импульса и массы жидкости получить асимптотически точное решение задачи для анализируемого потока; на базе полученного решения, в критеральной форме, исследовать зависимости кинетических характеристик потока от его критериев; обосновать адекватность полученных результатов расчета режимным параметрам действующего оборудования. В реализацию построенного в виде параметрически зависящего от критериальных параметров функционального ряда решения поставленной задачи о кинетике жидкостного потока предложен в форме итеративной процедуры алгоритм определения членов построенного ряда. На базе данного алгоритма проведено численное исследование с содержательным анализом поля скоростей и давления рассматриваемого потока по характерным для промышленного сепарирующего оборудования значениям критериальных параметров.
\end{abstract}

Ключевые слова: сепаратор, коническая вставка, вязкая несжимаемая жидкость, кинетика потока

\section{Введение}

Кинетические закономерности течения вязкой несжимаемой жидкости (ВНЖ) между двумя соосными близко отстоящими вращающимися ко- нусами, в том числе, и в межтарелочном зазоре (МT3) сепаратора имеют важное научно-техническое значение, что связано с проблемой количественного анализа как собственно гидродинамики этих потоков, так и с вытекающей отсюда оценкой 
эффективности процесса разделения суспензии в рабочем объеме машины. Где скомплектованные в виде яруса эквидистантные усеченные конуса (пакет тарелок) в роторе центробежного жидкостного сепаратора используются в качестве его основного рабочего органа (Рисунок 1).

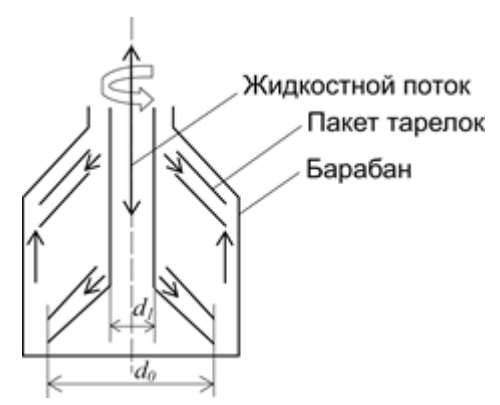

Рисунок 1. Конструктивная схема сепаратора-разделителя

Цель данной работы: на базе сформулированных для щелевого течения вязкой несжимаемой жидкости в полости между конусами соотношений в виде векторного уравнения Навье-Стокса (закона сохранения импульса) и уравнения неразрывности (закона сохранения массы жидкости) по анализируемому потоку, обосновать точное решение поставленной задачи. Используя полученное решение для данного течения, численным путем, исследовать кинетические зависимости от пространственных координат кинематических характеристик потока, давления в нем, мощности действующих на поток центробежных сил инерции и др. Результаты проведенного численного анализа проверить на адекватность их физическому смыслу исследуемого явления и соответствия полученных результатов параметрическим данным эксплуатируемых центробежных сепарирующих машин.

Исследованию особенностей режимов течения жидкости в М.З.сепаратора и связанных с этой проблемой задач посвящены работы (Александров, 2000, с. 24-29; Жуков, 1991, с. 747-751; Карпычев, 1982; Киряков, 2012, с. 46-49; Князев, 2011, с. 59-66; Пожарский, 2002; Романков, Плюшкин, 1976; Семенов, Славянский, Карамзин, 2014, с. 7-10; Семенов, 1997; 2017, Семенов, Славянский, Карамзин, 2017, с. 39-45; Карамзин, Семенов, 2012, с. 1619-1624; Кот, 1964, с. 227-237; Хаппель, Бреннер, 1976). Так, в статье (Жуков, 2016, с. 683-693) ставится и решается близкая к рассматриваемой в данной работе частная про- блема о нарушении сплошности потока однородной жидкостимежду тарелками центробежного сепаратора.

Аналогичные исследованным в указанных выше работах проблемы поднимались и при количественном анализе более простой задачи - о развитии течения В.Ж.в полости между двумя вращающимися равноотстоящими дисками (Аристов, Князев, 2012, с. 55-61; Шкоропад, 1975; Бэтчелор, 1973; Бликов, Рябчук, 2011; Нигматулин, 1987; Семенов, 1997, с. 55-63; Debuchy, Dument, Muhe, Micheau 1998, p. 791-810; Kreith, 1965, p. 11891190; Peube, Kreith, 1966, p. 261-286).

Хотя особенности однородных и двухфазных жидкостных систем в МТЗцентробежных разделяющих машин с коническими вставками изучались в ряде работ, однако не известен удобный для практического использования и адаптированный к современным информационным технологиям расчетный аппарат по моделированию циркулирующих потоков во вращающихся щелевых полостях конической формы. Не получили должного теоретического обоснования вопросы зависимости кинетики данного процесса от инерционной составляющей движения жидкости, оценки величины давления и мощности сил, действующих на поток и др.

В последние годы количественный анализ потоков жидкости, особенно в полостях сложной конфигурации, в ряде случаев проводился на основе таких компьютерных систем, как ANSYS, COSMOS Flo Works и др. В то же время полученные на базе этих систем результаты фактически эквивалентны данным разового численного эксперимента, с последующей адаптацией этих результатов на объект исследования. Что, по многим причинам, уступает полученным (когда такая возможность имеется) на основе явной или иной формах алгоритмов результатам прямого расчета (в том числе, вследствие их общности, удобства применения в прогнозировании протекания процесса, простоты использования и др.) $)^{1}$.

Поскольку тарелки сепаратора могут быть смоделированы коническими поверхностями в ортогональной биконической системе координат, то, таким образом, создаются предпосылки в корректной постановке и количественном моделировании, с достаточной точностью, краевой задачи по течению жидкости в МТ3. ${ }^{2}$

\footnotetext{
1 Кудрявцев Е.M. Mathcad 2000: символьное и численное решение разнообразных задач. М.: Д.К.Пресс, 2001. 571 с.

2 Касаткин А.Г. Основные процессы и аппараты химической технологии: учебник для вузов. 10-е изд. М.: Альянс, 2004.753 с.
} 
В связи с этим, основываясь на информационных технологиях, в работе предлагается более обоснованный, по сравнению с известными до сих пор аналогами, количественный анализ гидродинамики потока в полости между двумя моделирующими МТЗ близко отстоящими вращающимися конусами.

\section{Материалы и методы исследования}

ПустьО $\xi \varsigma \zeta$ - неподвижная прямоугольная декартова система координат с осью О , направленной вниз по оси вращения ротора (Рисунок 2).

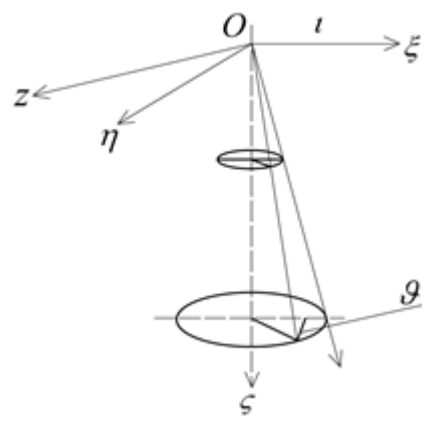

Рисунок 2. Схема к обоснованию биконической системы координат ( $\xi, \eta, \imath-$ подвижная прямоугольная декартова система координат, $r \vartheta z$ - биконическая система координат)

Задачу о течении жидкости между двумя безграничными конусами целесообразно относить к называемой биконической системе координат $\eta \vartheta z$, жестко связанной с одним из конусов (Рисунок 2). В качестве координатных поверхностей выбирается семейство двух взаимно ортогональных конусов $\eta=$ const, $z=$ const и осевая полуплоскость $\vartheta$ = const, с параметрами Ламе: $H \eta=1, H_{z}=1, H_{\vartheta}=$ $\eta \sin \alpha-z \cos \alpha$ (где $\alpha-$ полуугол конусности) (Карпычев, 1982).

В принятом допущении об узости зазора (Рисунок 3), вдали от оси О, очевидно, что ๆ $\sin \alpha$ много больше $z \cos \alpha$. В таком случае, если ротор вместе с системой координата $\rceil \vartheta z$ приведен во вращательное движение, то по скорости и ускорению жидкости $\partial / \partial z$ много больше $\partial / \partial \eta$.

Тогда, приближенно, при осесимметричном $(\partial / \partial \vartheta=0)$ и стационарном $(\partial / \partial t=0)$ режиме течения, если в качестве характерной длины выбиpать $l=(v / \Omega)^{1 / 2}$, характерной скорости $U=(v \cdot \Omega)^{1 / 2}($ где $v$ - кинематическая вязкость жидкости, $\Omega=\omega \sin \alpha$, $\omega$ - угловая скорость ротора), то в относительном движении в проекциях по осям $\eta, \vartheta, z$ система уравнений Навье-Стокса и уравнение неразрывности по- тока, в безразмерной форме, записываются в виде (Карпычев, 1982)

$$
\begin{aligned}
& u \frac{\partial u}{\partial r}+w \frac{\partial u}{\partial x}-\frac{1}{r} v^{2}= \\
& =-\frac{\partial P}{\partial r}+2 v+\frac{\partial^{2} u}{\partial x^{2}}+\frac{\partial^{2} u}{\partial r^{2}}+ \\
& +\frac{1}{r} \frac{\partial u}{\partial r}-\frac{\operatorname{ctg} \alpha}{r} \frac{\partial u}{\partial x}-\frac{1}{r^{2}} u+\frac{\operatorname{ctg} \alpha}{r^{2}} w,
\end{aligned}
$$

$u \frac{\partial v}{\partial r}+w \frac{\partial v}{\partial x}+\frac{u-w \operatorname{ctg} \alpha}{r} v=$

$$
=2(w \operatorname{ctg} \alpha-u)+\frac{\partial^{2} v}{\partial x^{2}}+\frac{\partial^{2} v}{\partial r^{2}}+
$$$$
+\frac{1}{r} \frac{\partial v}{\partial r}-\frac{\operatorname{ctg} \alpha}{r} \frac{\partial w}{\partial x}-\frac{1}{r^{2} \sin ^{2} \alpha} v
$$

$u \frac{\partial w}{\partial r}+w \frac{\partial w}{\partial x}+\frac{\operatorname{ctg} \alpha}{r} v^{2}=$

$=-\frac{\partial P}{\partial x}-2 v \operatorname{ctg} \alpha+\frac{\partial^{2} w}{\partial x^{2}}+\frac{\partial^{2} w}{\partial r^{2}}+$

$+\frac{1}{r} \frac{\partial w}{\partial r}-\frac{\operatorname{ctg} \alpha}{r} \frac{\partial w}{\partial x}+\frac{\operatorname{ctg} \alpha}{r^{2}} u-\frac{\operatorname{ctg}^{2} \alpha}{r^{2}} w$,

$\frac{\partial}{\partial r}(r u)+r \frac{\partial w}{\partial x}=0$

где $r=\eta / l ; x=z / l$ - соответственно - безразмерная продольная и поперечная координата; $u, v$, $w$ - соответственно - безразмерная продольная, относительная окружная и поперечная составляющая скорости жидкости, $\mathrm{P}=\mathrm{P}^{\prime} /\left(\rho U^{2}\right)$-безразмерное динамическое давление, $\mathrm{P}^{\prime}=p-\rho \omega^{2} \times$ $\times(\eta \sin \alpha-z \cos \alpha)^{2} / 2-$ динамическое давление (условно), $\mathrm{p}$ - давление, $\rho$ - плотность жидкости.

Асимптотически, при $\alpha \rightarrow \pi / 2$, полость между конусами трансформируется в горизонтальный диск, а система (1)-(4) вырождается в систему уравнений Навье-Стокса в цилиндрических координатах (Лойцянский, 1970).

Совмещая начало координат Ос образующей верхнего конуса и осью ротора, и направляя ось $z(\mathrm{x})$ вниз, ортогонально оси $\eta(r)$, в качестве граничных принимаем условия прилипания жидкости к стенкам канала (Рисунок 3)

$u=v=w=0$ при $\mathrm{x}=0$,

$u=v=w=0$ при $\mathrm{x}=\lambda, \lambda=h(\Omega / v)^{1 / 2}$,

где $\lambda$ - параметр Экмана (безразмерный зазор между конусами), $h$ - толщина МТ3 
Кроме того, должно быть выполнено условие

$u=0$ при $r \rightarrow \infty$,

где $u$ - модуль продольной скорости жидкости.

С учетом (7) составляющие скорости $u, v$ отыскиваются в виде рядов (Карпычев, 1982)

$u(r, \mathrm{x})=u_{1}(x) / r+u_{2}(x) / r^{2}+u_{3}(x) / r^{3}+\ldots$,

(8) Рисунок 3. Схема межтарелочной полости бараба-

$v(r, \mathrm{x})=v_{1}(x) / r+v_{2}(x) / r^{2}+v_{3}(x) / r^{3}+\ldots$

В свою очередь, в качестве динамического давления принимаем

$\mathrm{P}(r, \mathrm{x})=\mathrm{P}_{0}(\mathrm{x})+\mathrm{P}_{1}(\mathrm{x}) \ln r+\mathrm{P}_{2}(x) / r+\mathrm{P}_{3}(x) / r^{2}+\ldots$

Согласно (5), (6) для $u_{\mathrm{n}}, v_{\mathrm{n}}(n=1,2, \ldots)$ имеем граничные условия

$u_{\mathrm{n}}(\mathrm{x})=v_{\mathrm{n}}(\mathrm{x})=0$ при $\mathrm{x}=0$,

$u_{\mathrm{n}}(\mathrm{x})=v_{\mathrm{n}}(\mathrm{x})=0$ при $\mathrm{x}=\lambda$.

Условие расхода жидкости $q$ в безразмерном виде

$q=2 \pi \int_{0}^{\lambda} u(r, x) r \sin \alpha d x$,

где $q=Q U^{-1} l^{-2}=Q\left(\Omega / v^{3}\right)^{1 / 2}, q$ - параметр Россби, $Q$ расход жидкости через МТЗ

Учитывая соотношения (12), (13), для $u_{1}$ выбираем

$\int_{0}^{\lambda} u_{1}(x) d x=q /(2 \pi \sin \alpha)$

и тогда для $u_{\mathrm{n}}$ должны быть выполнены условия

$\int_{0}^{\lambda} u_{n}(x) d x=0, n=2,3, \ldots$

В свою очередь, интегрируя уравнение (4) по х, получим

$w=-\frac{1}{r} \frac{\partial}{\partial r} \int_{0}^{x} r u(r, x) d x$,

и поэтому, согласно (15), (16) граничные условия (5), (6) для поперечной составляющей $w$ скорости жидкости выполняются. В результате, принимая во внимание (8), (16), для данной составляющей имеем разложение по $r$, начинающееся с члена лишь порядка $\left(1 / r^{3}\right)$

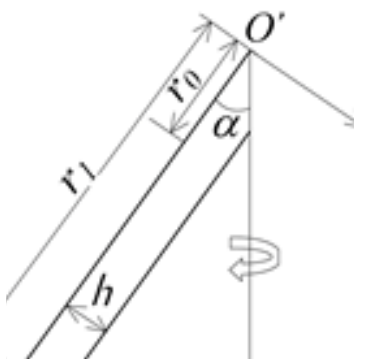

на сепаратора

$w(r, x)=\frac{1}{r^{3}} \int_{0}^{x} u_{2}(x) d x+\frac{2}{r^{4}} \int_{0}^{x} u_{3}(x) d x+\ldots$

Подставляя разложения (8)-(10), (17) в (1)-(3), и группируя члены при одинаковых степенях $r$, приходим к системе уравнений последовательных приближений:

- в нулевом приближении

$\mathrm{P}_{0}^{\prime}(\mathrm{x})=0, \mathrm{P}_{0}=\mathrm{C}_{0}, \mathrm{C}_{0}=0$,

поскольку на входе в канал давление составляет $\mathrm{p}_{0}=\rho \omega^{2} d_{0}^{2} / 8$ (Рисунок 1);

- по приближениям более высокого порядка:

$P_{1}^{\prime}(x)=0, P_{1}=C_{1} / 2, u_{1}^{\prime \prime}+2 v_{1}=$

$=C_{1} / 2, v_{1}^{\prime \prime}-2 u_{1}=0$;

$P_{2}^{\prime}(x)=-2 v_{1} \operatorname{ctg} \alpha, P_{2}(x)=$

$=-C_{2} / 2-2 \int_{0}^{x} v_{1} d x \operatorname{ctg} \alpha$

$u_{2}^{\prime \prime}+2 v_{2}=u_{1}^{\prime} \operatorname{ctg} \alpha-$

$-\mathrm{P}_{2}, v_{2}^{\prime \prime}-2 u_{2}=v_{1}^{\prime} \operatorname{ctg} \alpha$;

$P_{3}^{\prime}(x)=-2 v_{2} \operatorname{ctg} \alpha, P_{3}(x)=$

$=-C_{3} / 4-2 \int_{0}^{x} v_{2} d x \operatorname{ctg} \alpha$,

$u_{3}{ }^{\prime \prime}+2 v_{3}=u_{2}{ }^{\prime} \operatorname{ctg} \alpha-u_{1}^{2}-v_{1}^{2}-2 \mathrm{P}_{3}$,

и т.д.,

$v_{3}^{\prime \prime}-2 u_{3}=v_{2}^{\prime} \operatorname{ctg} \alpha+$

$+v_{1} \operatorname{ctg}^{2} \alpha-2 \operatorname{ctg} \alpha \int_{0}^{x} u_{2} d x$,

где штрихами обозначены производные по $x$. 
В соответствии с (17) для поперечной составляющей скорости жидкости будем иметь

$$
\begin{aligned}
& w(r, x)=\frac{1}{2 r^{3}}\left[v_{2}^{\prime}(x)-v_{2}^{\prime}(0)-\right. \\
& \left.-v_{1}(x) \operatorname{ctg} \alpha\right]+\frac{1}{r^{4}}\left[v_{3}^{\prime}(x)-v_{3}^{\prime}(0)-\right. \\
& \left.-v_{2}^{\prime}(0) \operatorname{ctg} \alpha \cdot x-2 \operatorname{ctg}^{2} \alpha \int_{0}^{x} v_{1} d x\right]+. .
\end{aligned}
$$

где входящие в (25) составляющие скорости $v_{1}, v_{2}, v_{3}$ находятся при решении систем уравнений (19)-(24).

Как видно, по своей структуре, (19), (20)-(21), (22)-(24), соответственно, представляют собой редуцированную систему трех обыкновенных дифференциальных уравнений, каждая из которых эквивалентна дифференциальному уравнению четвертого порядка по $v_{\mathrm{n}}$

$v_{\mathrm{n}}^{\mathrm{IV}}+4 v_{\mathrm{n}}=\mathrm{C}_{\mathrm{n}}+f_{\mathrm{n} 1-}(x), n=1,2, \ldots$

где $\mathrm{C}_{\mathrm{n}}$ - постоянные величины, $f_{\mathrm{n} 1-}$ - выражения, зависящие от низших приближений.

Общее решение уравнения (26) представляется в виде ${ }^{3}$

$$
\begin{aligned}
& v_{\mathrm{n}}(x)=C_{\mathrm{n} 1} y_{1}(x)+\ldots+C_{\mathrm{n} 4} \mathrm{y}_{4}(x)+ \\
& \left.+C_{\mathrm{n}}\left[1-y_{1}(\mathrm{x})\right] / 4+\int_{0}^{x} y_{4}(x-\xi) f_{n-1}(\xi)\right] d \xi,
\end{aligned}
$$

где $\mathrm{y}_{1}=\operatorname{ch} x \cdot \cos x, \mathrm{y}_{2}=(\operatorname{ch} x \cdot \sin x+\operatorname{sh} x \cdot \cos x) / 2$, $\mathrm{y}_{3}=\operatorname{sh} x \cdot \sin x / 2, \mathrm{y}_{4}=(\operatorname{ch} x \cdot \sin x-\operatorname{sh} x \cdot \cos x) / 4-$ функции А.Н. Крылова.

В силу (11) $\mathrm{C}_{\mathrm{n} 1}=\mathrm{C}_{\mathrm{n} 3}=0$, и в результате вместо (27) имеем

$$
\begin{aligned}
& v_{\mathrm{n}}=C_{\mathrm{n} 2} y_{2}(x)+C_{\mathrm{n} 4} y_{4}(x)+ \\
& \left.+C_{\mathrm{n}}\left[1-y_{1}(\mathrm{x})\right] / 4+\int_{0}^{x} y_{4}(x-\xi) f_{n-1}(\xi)\right] d \xi .
\end{aligned}
$$

Поэтому согласно (14), (19) коэффициенты $C_{12}, C_{14}$, $C_{1}$ по первому приближению определяются как решение системы трехлинейных неоднородных алгебраических уравнений

$$
\left\{\begin{array}{l}
\mathrm{y}_{2} \mathrm{C}_{12}+\mathrm{y}_{4} \mathrm{C}_{14}+0,25\left(1-\mathrm{y}_{1}\right) \mathrm{C}_{1}=0, \\
-4 \mathrm{y}_{4} \mathrm{C}_{12}+\mathrm{y}_{2} \mathrm{C}_{14}+\mathrm{y}_{3} \mathrm{C}_{1}=0 \\
\mathrm{C}_{12}\left(\mathrm{y}_{1}-1\right)+\mathrm{y}_{3} \mathrm{C}_{14}+\mathrm{y}_{4} \mathrm{C}_{1}=q /(\pi \sin \alpha),
\end{array}\right.
$$

где использованы зависимости

$$
\begin{aligned}
& y_{1}^{\prime}=-4 y_{4}, y_{2}^{\prime}=y_{1}, y_{3}^{\prime}=y_{2}, y_{4}^{\prime}= \\
& =y_{3} ; y_{1}^{\prime \prime}=-4 y_{3}, y_{2}^{\prime \prime}=-4 y_{4}, y_{3}^{\prime \prime}= \\
& =y_{1}, y_{4}^{\prime \prime}=y_{2},
\end{aligned}
$$

и, по умолчанию, принято $y_{\mathrm{k}}=y_{\mathrm{k}}(\lambda), k=1,2,3,4$.

В свою очередь, коэффициенты $C_{\mathrm{n} 2}, C_{\mathrm{n} 4}, C_{\mathrm{n}}(n=2$, $3, .$.$) отыскиваются аналогично на базе алгебра-$ ической системы с тем же детерминантом левой части, что и в (29), но заданными в форме

$$
\begin{aligned}
& \left.\left.\int_{0}^{\lambda} y_{4}(x-\xi) f_{n-1}(\xi)\right] d \xi, \int_{0}^{\lambda} y_{2}(x-\xi) f_{n-1}(\xi)\right] d \xi \\
& \left.\int_{0}^{\lambda} y_{3}(x-\xi) f_{n-1}(\xi)\right] d \xi \text { правыми частями. }
\end{aligned}
$$

Где, например, повторому и третьему приближениям

$f_{1}(x)=\left(v_{1}^{I I I}+2 u_{1}^{I}+4 \int_{0}^{x} v_{1} d x\right) \operatorname{ctg} \alpha=$

$=\left(v_{1}^{I I I}+C_{1} x\right) \operatorname{ctg} \alpha$,

$$
\begin{aligned}
& f_{2}(x)=\left(v_{2}^{I I I}+8 \int_{0}^{x} v_{2} d x\right) \operatorname{ctg} \alpha+ \\
& +v_{1}^{I I} \operatorname{ctg}^{2} \alpha-2\left(u_{1}^{2}+v_{1}^{2}\right) .
\end{aligned}
$$

Полученные согласно (28) зависимости для окружной скорости потока используются при определении действующего на поток крутящего момента со стороны конусов и мощности от этого момента. При расчете крутящего момента учитывается, что на единицу площади вышележащего конуса со стороны потока действует сила вязкостного трения

$p_{z} \vartheta=\rho v \partial u \vartheta / \partial z$ при $z=0$,

где $u \vartheta$ - размерная относительная окружная скорость частицы жидкости, или, согласно (9), в безразмерной форме

$\mathrm{p}_{\mathrm{x}} \vartheta=v^{\prime}(0)$

где $p_{x} \vartheta-$ безразмерное касательное напряжение.

В таком случаесогласно (32) на кольцевой элемент конуса с образующей $\mathrm{d} r$ и площадью $2 \pi r \mathrm{~d} r$ действует крутящий момент

\footnotetext{
3 Камке Э. Справочник по обыкновенным дифференциальным уравнениям. 4-е изд., испр. М.: Наука, 1971. 576 с.
} 
$\mathrm{d} M=2 \pi(\partial v / \partial x) r^{2} \mathrm{~d} r \sin ^{3} \alpha$, при $x=0$

или, с учетом (9), сдостаточной точностью,

$\mathrm{d} M=2 \pi\left[v_{1}^{\prime}(0) r+v_{2}{ }^{\prime}(0)\right] d r \sin ^{3} \alpha$.

Откуда, интегрируя по $r$, получим (в размерных переменных)

$$
\begin{aligned}
& M=2 \pi\left[v_{1}^{\prime}(0)\left(r_{1}^{2}-r_{0}^{2}\right)+2 v_{2}^{\prime}(0)\left(r_{1}-r_{0}\right)\right] \times \\
& \times \rho \cdot \sqrt{v^{5} / \Omega} \cdot \sin ^{3} \alpha,
\end{aligned}
$$

где $r_{0}=\eta_{0} / l, r_{1}=\eta_{1} / l-$ соответственно, наименьший и наибольший продольной размер тарелки (Рисунок 3), а также мощность момента

$N=M \cdot \omega$,

где $M$ определяется по (33).

В свою очередь, приближенно, согласно (10) давление на выходе

$\mathrm{p}_{1}=\mathrm{p}_{1}\left(r_{1} \cdot l\right)=\mathrm{P}_{0}^{\prime}+\left(r_{1}^{2} / 2+\mathrm{P}_{1} \ln r_{1}\right) \cdot \rho U^{2}$,

где в соответствии с (18), (19) $\mathrm{P}_{0}^{\prime}=0, \mathrm{P}_{1}=\mathrm{C}_{1} / 2$.

\section{Результаты \\ и их обсуждение}

В качестве объекта исследования выбирался сепаратор Ж5-ОСЦП-1. Конструктивные и режимные параметры машины: число тарелок - 63; полуугол конусноститарелки $\alpha=40^{\circ}$; минимальный и максимальный диаметр тарелки, соответственно, $d_{0}=0,066, d_{1}=0,175 \mathrm{M}$, толщина МТ3 $-h=0,4 \mathrm{MM}$ (Рисунок 1); производительность $Q=1 \mathrm{~m}^{3} /$ час; угловая скорость ротора $\omega=837$ рад/с; кинематическая вязкость жидкости (молока) $-v=1,8 \cdot 10^{-}$ ${ }^{6} \mathrm{M}^{2} / \mathrm{c}$, плотность $\rho=1030 \mathrm{\kappa г} / \mathrm{M}^{3}$.

При численном анализе составляющих скорости потока по зависимостям (8), (9), (30), (31) ограничивались четырьмя членами в разложениях рядов, в (25) - двумя членами.

На базе близких для реальных условий значений критериальных параметров $\lambda$ (безразмерной толщины МТЗ и $q$ (безразмерного расхода) приведены отраженные графиками результаты количественного анализа кинематики потока на относительно небольшом (порядка $15 \lambda$ ) и сравнительно удаленном (порядка $30 \lambda$ ) расстоянии $r$ от оси вращения (Рисунки 4-6).
Следует отметить, что в быстро вращающемся роторе ограниченный стенками МТЗ жидкостной поток в связанной с ротором подвижной системе отсчета подвержен действию на него силового поля значительной (по сравнению с силой тяжести) интенсивности массовых сил инерции: переносной, Кориолиса и Даламбера, а также поверхностных сил - давления и внутреннего трения (Лойцянский, 1970).

Как показывает численный анализ, влияние данных силовых факторов на движение потока существенно различается при разном удалении от оси вращения ротора (Рисунки 4-6).

Известно, что для приближенного по геометрической структуре к рассматриваемому в работе потоку между дисками, вследствие предполагае-

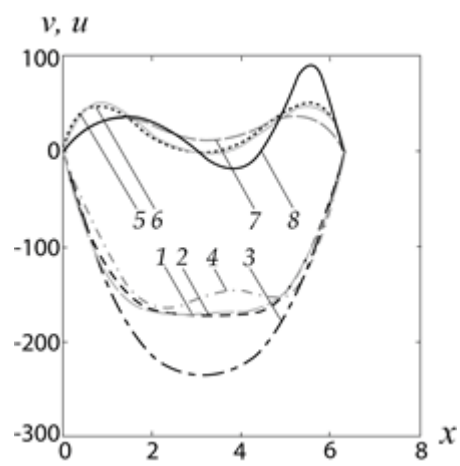

Рисунок 4. Профили безразмерных относительной окружной $v$ и продольной скорости $u$ жидкости при значениях режимных параметров процесса $\lambda=2 \pi, q=10^{5} ; r=15 \lambda$ (по скоростиv: 1 - 1-ое, 2 2-ое, 3 - 3-е, 4 - 4-ое приближение; по скорости u: 5 - 1-ое, 6 - 2-ое, 7 - 3-е, 8 - 4-ое приближение)

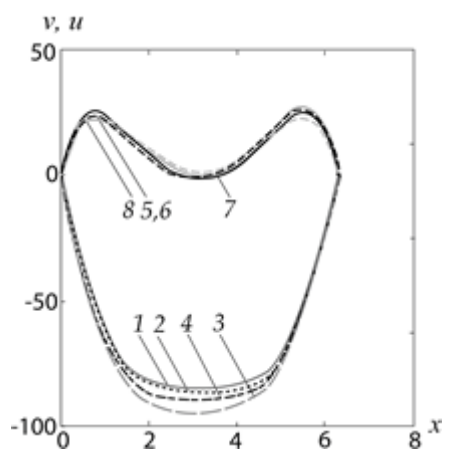

Рисунок 5. Профили безразмерных относительной окружной $v$ и продольной скорости $u$ жидкости при значениях режимных параметров процесса $\lambda=2 \pi, q=10^{5} ; r=30 \lambda$ (по скоростиv: 1 - 1-ое, 2 2-ое, 3 - 3-е, 4 - 4-ое приближение; по скорости u: 5 - 1-ое, 6 - 2-ое, 7 - 3-е, 8 - 4-ое приближение) 


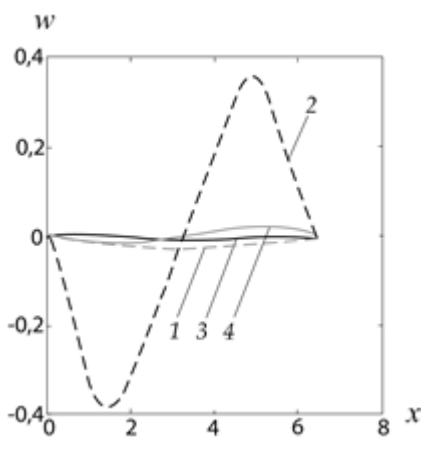

Рисунок 6. Профиль поперечной скорости $w$ жидкости $\left(\lambda=2 \pi, q=10^{5} ; r=15 \lambda: 1\right.$ - 1-ое, 2 - 2-ое приближение; $r=30 \lambda: 3$ - 1-ое, 4 - 2-ое приближение)

мой симметрии поля скоростей относительно середины зазора, слагаемые с четными номерами в разложениях решения для диска, аналогичные (8), (9), а вследствие (8), (17) - для поперечной скорости $w$, с нечетными номерами, выпадают (Kreith, 1965, p. 1189-1190; Семенов, 1997, с. 55-63; Славянский, 2007). В результате чего вклад в решение собственно по величине второй, базирующийся на решении линейной системы (21) итерации, для течения между конусами, рассматриваемого как поправка ко второй (равной нулю) итерации для диска, оказывается относительно небольшим (порядка $2 \%$ по относительной окружной $v$ и $4 \%$ по продольной $u$ составляющим скорости потока). Поэтому, как и в случае диска, графики 1 и 2, 5 и 6, Рисунки 4, 5 практически симметричны относительно середины МТ3

Анализ базирующихся на втором (линейном) приближении разложений (8), (9) графиков 2 и 6 рисунков 4, 5 выявляет особенности кинематических характеристик потока как результат воздействия на него (в пренебрежении силой Даламбера) градиента $\partial \mathrm{P} / \partial r$ динамического давления, силы Кориолиса и вязкостной силы.

Так, в силу линейности краевых задач (11), (12), (19), (21),через посредство числа $q$ Россби (13), при фиксированном $r$, градиентом $\partial \mathrm{P} / \partial r$ обеспечивается геометрическое подобие профилей для скоростей $v$ и $u$, собственно, по первому и второму членам разложений (8) и (9).

Анализ графиков 1,2 и 5, 6, рисунков 4, 5, с точки зрения их формы, свидетельствует о взаимном «упругом» влиянии на кривизну этих линий окружной и продольной составляющих силы Кориолиса. Поскольку, в соответствии с выбранными значениями параметров $\lambda$ и q, согласно данным этих графиков, вследствие большой закрутки потока вблизи оси вращения ротора, окружная скорость по абсолютной величине значительно превышает расходную скорость u потока, то относительная окружная скорость $\mathrm{v}$, совместно с угловой скоростью $\omega$ ротора, индуцирует направленную против u поворотную силу. Что обусловливает замедление потока в его ядре при движении жидкости к периферии канала. В свою очередь, продольная составляющая скорости u также, но с меньшим эффектом, оказывает через воздействие на профиль скорости в окружном направлении составляющей силы Кориолиса «упругое» тормозящее влияние на поток в его ядре.

С другой стороны, из сравнения габитуса графиков 1-3 и 4, а также Графиков 5-7 и 8, Рисунок 4, можно заключить, что вблизи от входа в канал (на расстоянии порядка $r_{1}=15 \lambda$ ) наибольшее влияние на структуру профилей скоростей $v$ и $u$ оказывает обусловленная конвективной составляющей движения сила Даламбера. В том числе, и от действия этой силы в продольном направлении вблизи нижней тарелки, (при $\mathrm{x}=2 \pi)$ и в окресности середины зазора (при $\mathrm{x}=\pi)$ отмечаются зоны возвратного течения, с основным потоком жидкостив направлении перифериитарелки в интервале между двумя этими зонами.

По той же причине, вблизи от входа в канал, зависящее собственно от первых двух приближений $v_{1}$ и $v_{2}$ первое слагаемое в формуле (25) для $w$ также мало (кривая 1, рис. 6), и вид профиля $w$ определяется, в основном, связанной через (23) с конвективной компонентой движения потока составляющей скорости $v_{3}$ (кривая 2 , рисунок 6).

Поскольку, согласно закону сохранения массы (4), и в соответствии с графиком 2 Рисунок 6, профиль скорости w относительно середины МТЗ имеет антисимметричный вид, то это показывает, что жидкость в канале отбрасывается как к нижней, так и к верхней стенкам его. В частности, отсюда следует, что в двухфазном жидкостном потоке влиянием поперечной составляющей скорости на процессе диментации взвешенных в потоке твердых частиц можно пренебречь.

С удалением от входа в канал возмущающее влияние на кинетику потока силы инерции Даламбера убывает, баланс силовых факторов - градиента динамического давления, поворотной силы Кориолиса и внутреннего трения - выравнивается, в результате чего габитусы профилей $\mathrm{u}, \mathrm{v}, \mathrm{w}$, по последовательным итерациям, становятся мало различимыми (графики кривых Рисунок 5, кривых 3 , 4, Рисунок 6). Что, в плане обоснованности при- 
нятой при количественном анализе кинематики исследуемого режима течения расчетной схемы в форме краевой задачи (1)-(7), и ее решения с помощью рядов (8), (9), (17), в рамках выбранных значений критериальных величин $\lambda$ и q, свидетельствует о сходимости построенного итерационного процесса на достаточно большом удалении от оси ротора.

Для получения надежных данных по профилям скоростей течения жидкости на близком (не превышающем пятнадцати зазоров канала) расстоянии от входа в канал необходимо проводить расчеты по пятому, шестому или большему числу итераций. Однако, хотя на проведение расчетов на ПК по четырем приближениям затрачивается и незначительное (порядка 10") время, численное моделирование на базе предложенного алгоритма, из-за обусловленных нелинейностью правых частей в высших итерациях кратных квадратур все более высокого порядка, приводит к трудоемким и длительным вычислениям.

В свою очередь, на расстоянии, превышающем толщину канала в тридцать раз, можно ограничиться меньшим числом итераций.

С точки зрения анализа динамики потока нужно отметить, что отраженные графиками (Рисунок 7) результаты расчета давления по выражению (35) выявляют экспоненциальный рост этого показателя в канале при удалении от оси ротора (кривые 1,2$)$ и убывание давления, когда расход жидкости растет (кривая 2 ниже кривой 1). Что, применительно к расходящемуся режиму течения, вытекает из закона сохранения энергии.

На базе зависимостей (33), (34) для сепаратора Ж5-ОСЦП-1 рассчитали крутящий момент $M=0,02 \mathrm{H} \cdot \mathrm{M}$ и полезную мощность двигателя

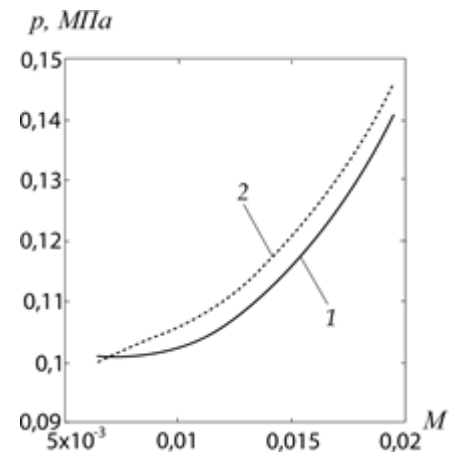

Рисунок 7. Зависимости давления $p$ (МПа) жидкости от продольной координаты $r(\mathrm{M})$ и безразмерного расхода $q\left(\lambda=2 \pi: 1-q=10^{4}, 2-q=10^{5}\right)$
$N=1,06$ кВт. Поскольку номинальная мощность двигателя 1,5 кВт, то полученное расчетное значение мощности следует считать близким номинальной. Пониженная, по сравнению с номинальной мощностью, величина этого показателя объясняется тем, что, кроме затрачиваемой на вращение ротора полезной работы, энергия двигателя расходуется на поддержание жидкостного напора, а также работу по преодолению внешних сил сопротивления (трения в подшипниках, трения воздушного потока о ротор и др.). Полученные данные свидетельствуют об их непротиворечивости и согласии с физическим смыслом результатов в целом количественного анализа гидродинамики исследуемого щелевого потока.

\section{Выводы}

Таким образом, в рамках сформулированной на базе законов сохранения импульса и массы краевой задачи для тонкослойного циркулирующего потока В.Ж.от линейного источника на оси двух эквидистантных вращающихся с постоянной частотой вращения конусов предлагается универсальный алгоритм для расчета гидродинамических характеристик исследуемого течения. Количественным анализом выявлены необходимые для инженерных расчетов особенности кинематики течения, расхода и давления жидкости. На конкретном примере машины рассчитывается мощность действующего на поток крутящего момента, с обоснованием согласия результатов расчетов по номинальной мощности двигателя.

\section{Литература}

Александров О.Е. Идеальная центрифуга // Журнал технической физики. 2000. Т. 70, № 9. с. 24-29.

Аристов С.Н., Князев Д.В. Течения вязкой жидкости между подвижными параллельными плоскостями // Известия РАН Механика жидкости и газа. 2012. № 4. с. 55-61.

Блинов Д., Рябчук г. Математическое моделирование процесса нанесения двухслойной оболочки: математическое моделирование процесса нанесения двухслойной оболочки на сферические гранулы в центробежном поле. L.P.Lambert Academic Publishing, 2011. 148 с. (Russian Edition)

Бэтчелор Д. Введение в динамику жидкости / пер. с англ. Д. Бэтчелор. М.: Мир, 1973. 758 с.

Жуков В.Г. Центробежная радиальная фильтрация в условиях переменной проницаемости пористого осадка // Теоретические основы химической технологии. 1991. Т. 25, № 6. с. 747-751. 
Жуков В.Г., Чесноков В.М. Давление в тонкослойном потоке жидкости тарельчатого центробежного сепаратора // Теоретические основы химической технологии. 2016. Т. 50, № 6. с. 683693. https://doi.org/10.7868/S004035711606021Х

Карамзин А.В., Семенов Е.В. Количественный анализ процесса фракционирования тонкодисперсных частиц в центробежном сепараторе // Журнал прикладной химии. 2012. Т. 85, № 10. с. $1619-1624$.

Карпычев В.А., Семенов Е.В. Гидромеханические процессы технологической обработки молочных продуктов. М.: Легкая и пищевая промышленности, 1982. 240 с.

Киряков С.И., Митрофанов Ю.А. Специальное центробежное оборудование для разделения жидких неоднородных сред // Цветные металлы. 2012. № 1. с. 46-49.

Князев Д.В. Осесимметричные течения несжимаемой жидкости между подвижными вращающимися дисками // Известия РАН Механика жидкости и газа. 2011. № 4. с. 59-66.

Кот Ю.Д. Математические зависимости процесса центрифугирования утфелей // Труды В.И.СП. 1964. Вып. 12. с. 227-237.

Лойцянский Л.Г.Механика жидкости и газа. М.: Наука, 1970. 823 с.

Нигматулин Р.И. Основы механики многофазных смесей. М.: Наука, 1987. Ч. 2. 464 с.

Пожарский Ю.М. Разработка и математическое моделирование центробежного дискового сепаратора на постоянных магнитах / автореф. на соиск. ученой степ. канд. техн. наук: 05.05.06 горные машины. Владикавказ, 2002. 17 с.
Романков П.Г., Плюшкин С.А. Жидкостные сепараторы. Л.: Машиностроение, 1976. 328 с.

Семенов Е.В. К обоснованию асимптотического решения для ламинарного течения жидкости между двумя вращающимися дисками // Прикладная механика и техническая физика. 1997. Т. 38, № 3. с. 55-63.

Семенов Е.В., Славянский А.А., Карамзин В.А. Количественное моделирование процесса разделения суспензий в роторе фильтрующей центрифуги периодического действия // Химическое и нефтегазовое машиностроение. 2014. № 11. c. 7-10.

Семенов Е.В., Славянский А.А., Карамзин А.В. К расчету гидродинамических характеристик тарельчатого сепаратора // Хранение и переработка сельхозсырья. 2017. № 6. с. 39-45.

Хаппель Д., Бреннер г. Гидродинамика при малых числах Рейнольдса. М.: Мир, 1976. 630 с.

Шкоропад Д.Е. Центрифуги для химических производств. М.: Машиностроение, 1975. 248 с.

Debuchy R., Dument A., Muhe H., Micheau P. Radial inflow between a rotating and stationary disc // European Journal of Mechanics - B.F.uids. 1998. Vol. 17, issue 6. P. 791-810. https://doi. org/10.1016/S0997-7546(99)80014-4

Kreith F. Reverse transition in radial source flow between two parallel planes // The Physics of Fluids. 1965. Vol. 8, issue 6. P. 1189-1190. https:// doi.org/10.1063/1.1761374

Peube J.-L., Kreith F. L'ecoulement permanent d'un fluidevisqueux incompressible entre deuxdisquesparallelesen rotation // Journal de Mécanique. 1966. Vol. 5. P. 261-286. 


\title{
About the Fluid Flow in the Centrifugal Separator
}

\author{
Anatoliy A. Slavyanskiy \\ Moscow State University of Technology and Management named after K.G. Razumovsky \\ (the First Cossacs University) \\ 73, Zemlyanoy Val str., Moscow, 109004, Russian Federation \\ E-mail: slavyanskiyaa@mgutm.ru
}

Evgeniy V. Semenov
Moscow State University of Technology and Management named after K.G. Razumovsky
(the First Cossacs University)
73, Zemlyanoy Val str., Moscow, 109004, Russian Federation
7 7E-mail: sem-post@mail.ru

Vera A. Gribkova

Moscow State University of Technology and Management named after K.G. Razumovsky

(the First Cossacs University)

73, Zemlyanoy Val str., Moscow, 109004, Russian Federation

E-mail:vera_gribkova@list.ru

Natalia V. Nikolaeva

Moscow State University of Technology and Management named after K.G. Razumovsky

(the First Cossacs University)

73, Zemlyanoy Val str., Moscow, 109004, Russian Federation

E-mail:nata_nik@inbox.ru

\begin{abstract}
Features of the flow modes of viscous incompressible fluid in the cavity between the two rotating cones make a big difference due to the importance of the problem of analyzing the characteristics of the kinetics of these streams for centrifugal separating equipment in the dairy, meat, microbiological and other related industries. It is obvious that to study the kinetic patterns of fluid movement in a narrow gap between the two neighboring truncated cones it is necessary to have a correct apparatus of mathematical modeling of the current in this gap, bearing in mind that the flow induced by a linear source on the rotor rotation axis approximates the flow of the loosely concentrated system «liquid + solid» in the slit cavity between the separator plates. The aim in this paper: based on the laws of maintaining the pulse and mass of the liquid to obtain an asymptomatically accurate solution to the problem for the analyzed flow; on the basis of the received solution, in a criminal form, to investigate the dependence of kinetic characteristics of the flow from the current criteria; to justify the adequacy of the results of the calculation to the regime parameters of the operating equipment. In the implementation of the solution of the problem of the kinetics of a liquid flow, constructed in the form of a parametrically dependent on the criterial parameters of the functional series, an algorithm for determining the members of the constructed series is proposed in the form of an iterative procedure. On the basis of this algorithm, a numerical study was carried out with a meaningful analysis of the velocity and pressure field of the flow under consideration according to the values of the criterion parameters characteristic of industrial separation equipment.
\end{abstract}

Keywords: separator, tapered insertion, viscous incompressible liquid, kinetics of the stream.

\section{References}

Aleksandrov O.E. Ideal'naya tsentrifuga [The ideal centrifuge]. Zhurnal tekhnicheskoi fiziki [Journal of technical physics], 2000, vol. 70, no. 9, pp, 24-29.

Aristov S.N., Knyazev D.V. Techeniya vyazkoi zhidkosti mezhdu podvizhnymi parallel'nymi ploskostya- mi [Flow of viscous fluid between moving parallel planes]. Izvestiya R.N. Mekhanika zhidkosti i gaza [News of the Russian Academy of Sciences. Fluid and gas mechanics], 2012, no. 4, pp. 55-61.

Blinov D., Ryabchuk G. Matematicheskoe modelirovanie protsessa naneseniya dvukhsloinoi obolochki: matematicheskoe modelirovanie protsessa 
naneseniya dvukhsloinoi obolochki na sfericheskie granuly $\mathrm{v}$ tsentrobezhnom pole [Mathematical modeling of the two-layer coating process]. LAP. Lambert Academic Publishing, 2011, 148 p.

Betchelor D. Vvedenie $\mathrm{v}$ dinamiku zhidkosti [Introduction to fluid dynamics]. Moscow: Mir, 1973.758 p.

Zhukov V.G. Tsentrobezhnaya radial'naya fil'tratsiya $\mathrm{v}$ usloviyakh peremennoi pronitsaemosti poristogo osadka [Centrifugal radial filtration under conditions of variable permeability of porous sediment]. Teoreticheskie osnovy khimicheskoi tekhnologii [Theoretical foundations of chemical technology], 1991, vol, 25, no. 6, pp. 747-751.

Zhukov V.G., Chesnokov V.M. Davlenie v tonkosloinom potoke zhidkosti tarel'chatogo tsentrobezhnogo separatora [Pressure in the thin-layer liquid flow of the disc centrifugal separator]. Teoreticheskie osnovy khimicheskoi tekhnologii [Theoretical foundations of chemical technology], 2016, vol. 50, no. 6, pp. 683693. https://doi.org/10.7868/S004035711606021X

Karamzin A.V., Semenov E.V. Kolichestvennyi analiz protsessa fraktsionirovaniya tonkodispersnykh chastits $\mathrm{v}$ tsentrobezhnom separatore [A quantitative analysis of the fractionation of fine particulate matter in a centrifugal separator]. Zhurnal prikladnoi khimii [Journal of Applied Chemistry], 2012, vol. 85, no. 10, pp. 1619-1624.

Karpychev V.A., Semenov E.V. Gidromekhanicheskie protsessy tekhnologicheskoi obrabotki molochnykh produktov [Hydro-mechanical processes of technological processing of dairy products]. Moscow: Legkaya i pishchevaya promyshlennosti, $1982.240 \mathrm{p}$.

Kiryakov S.I., Mitrofanov Yu.A, pppetsial'noe tsentrobezhnoe oborudovanie dlya razdeleniya zhidkikh neodnorodnykh sred [Special centrifugal equipment for separation of liquid inhomogeneous media]. Tsvetnye metally [Nonferrous metal], 2012, no. 1, pp. 46-49.

Knyazev D.V. Osesimmetrichnye techeniya neszhimaemoi zhidkosti mezhdu podvizhnymi vrashchayushchimisya diskami [Axisymmetric flow of an incompressible fluid between rotating disks moving]. Izvestiya R.N. Mekhanika zhidkosti i gaza [News of the Russian Academy of Sciences. Fluid and gas mechanics], 2011, no. 4, pp. 59-66.

Kot Yu.D. Matematicheskie zavisimosti protsessa tsentrifugirovaniya utfelei [Mathematical dependences of the process of centrifugation of wafers]. Trudy V.I.SP [Proceedings of the all-union scientific research institute of agricultural products], 1964, vol. 12, pp. 227-237.

Loitsyanskii L.G.Mekhanika zhidkosti i gaza [Fluid and gas mechanics]. Moscow: Nauka, 1970. 823 p.

Nigmatulin R.I. Osnovy mekhaniki mnogofaznykh smesei [Fundamentals of mechanics of multi- phase mixtures]. Moscow: Nauka, 1987. Part 2. $464 \mathrm{p}$.

Pozharskii Yu.M. Razrabotka i matematicheskoe modelirovanie tsentrobezhnogo diskovogo separatora na postoyannykh magnitakh. Avtopef. diss. kand. tekhn. nauk. [Development and mathematical modeling of a centrifugal disk separator on permanent magnets. Abstract of Ph.D. (Technical) thesis]. Vladikavkaz, 2002. 17 p.

Romankov P.G., Plyushkin S.A. Zhidkostnye separatory [Liquid separators]. Leningrad: Mashinostroenie, 1976. $328 \mathrm{p}$.

Semenov E.V. K obosnovaniyu asimptoticheskogo resheniya dlya laminarnogo techeniya zhidkosti mezhdu dvumya vrashchayushchimisya diskami [To substantiate an asymptotic solution for laminar fluid flow between two rotating disks]. Prikladnaya mekhanika i tekhnicheskaya fizika [Applied mechanics and technical physics], 1997, vol. 38, no. 3, pp. 55-63.

Semenov E.V., Slavyanskii A.A., Karamzin V.A. Kolichestvennoe modelirovanie protsessa razdeleniya suspenzii v rotore fil'truyushchei tsentrifugi periodicheskogo deistviya [Quantitative modeling of the suspension separation process in the rotor of a batch filter centrifuge]. Khimicheskoe i neftegazovoe mashinostroenie [Chemical and oil and gas engineering], 2014, no. 11, pp. 7-10.

Semenov E.V., Slavyanskii A.A., Karamzin A.V. K raschetu gidrodinamicheskikh kharakteristik tarel'chatogo separatora [To calculate the hydrodynamic characteristics of the plates separator]. Khranenie $i$ pererabotka sel'khozsyr'ya [Storage and processing of agricultural raw materials], 2017, no. 6, pp. 39-45.

Khappel' D., Brenner G. Gidrodinamika pri malykh chislakh Reinol'das [Hydrodynamics at small Reynolds numbers]. Moscow: Mir, 1976. 630 p.

Shkoropad D.E. Tsentrifugi dlya khimicheskikh proizvodstv [Centrifuges for chemical production]. Moscow: Mashinostroenie, 1975, 248 p.

Debuchy R., Dument A., Muhe N., Micheau P. Radial Inflow Between a Rotating and Stationary Disc. European Journal of Mechanics - B.F.uids. 1998, vol. 17, issue 6, pp. 791-810. https://doi.org/10.1016/ S0997-7546(99)80014-4

Kreith F. Reverse Transition in Radial Source Flow Between Two Parallel Planes. The Physics of Fluids, 1965, vol. 8, issue 6, pp. 1189-1190. https://doi. org/10.1063/1.1761374

Peube J.-L., Kreith F. L'ecoulement Permanent D'un Fluidevisqueux Incompressible Entre Deuxdisquesparallelesen Rotation [The Permanent Flow of an Incompressible Viscous Fluid Between Two Rotating Parallel Discs]. Journal de Mécanique [Mechanics Journal], 1966, vol. 5, pp. 261-286. 